\title{
Population-based incidence of lymphoid neoplasms: Twenty years of epidemiological data in the Girona province, Spain
}

\author{
Marta Solans ${ }^{\mathrm{a}, \mathrm{b}, \mathrm{c}, *}$, Anna Fàbrega ${ }^{\mathrm{c}}$, David Morea ${ }^{\mathrm{c}}$, Carmen Auñon-Sanz ${ }^{\mathrm{d}}$, Isabel Granada ${ }^{\mathrm{e}}$, \\ Josep Maria Roncero ${ }^{\mathrm{f}}$, Antonio Blanco ${ }^{\mathrm{f}}$, Nicholas Kelleher ${ }^{\mathrm{f}}$, Joan Buch ${ }^{\mathrm{f}}$, Marc Saez ${ }^{\mathrm{a}, \mathrm{b}}$, \\ Rafael Marcos-Gragera ${ }^{\text {a,b,c }}$ \\ ${ }^{a}$ Centro de Investigación Biomédica en Red: Epidemiología y Salud Pública (CIBERESP), Av. Monforte de Lemos 3-5, 28029 Madrid, Spain \\ ${ }^{\mathrm{b}}$ Research Group on Statistics, Econometrics and Health (GRECS), University of Girona, C/ Universitat de Girona 10, 17003 Girona, Spain \\ ${ }^{\mathrm{c}}$ Epidemiology Unit and Girona Cancer Registry, Oncology Coordination Plan, Catalan Institute of Oncology, Carrer del Sol 15, 17004 Girona, Spain \\ ${ }^{\mathrm{d}}$ Radiation Oncology Service, Josep Trueta University Hospital, Catalan Institute of Oncology, Avinguda de França, S/N, 17007 Girona, Spain \\ ${ }^{\mathrm{e}}$ Hematological Laboratory Service, Germans Trias i Pujol University Hospital, Catalan Institute of Oncology, Josep Carreras Leukaemia Research Institute, Ctra. de Can \\ Ruti Camí de les Escoles S/N, 8916 Badalona, Spain \\ ${ }^{\mathrm{f}}$ Hematological Service, Josep Trueta University Hospital, Catalan Institute of Oncology, Avinguda de França, S/N, 17007 Girona, Spain
}

\section{A R T I C L E I N F O}

\section{Keywords:}

Lymphoid neoplasms

Population-based

Incidence

WHO 2008

Spain

\begin{abstract}
A B S T R A C T
Background: The aim of this study was to describe incidence patterns of lymphoid neoplasms in the Girona province (Spain) (1996-2015), and to predict the number of cases in Spain during 2020.

Methods: Data were extracted from the Girona cancer registry. Incident cases were classified using the ICD-O-3, third revision, and grouped according to the WHO 2008 classification scheme. Age-adjusted incidence rates to the European standard population (ASRE) were estimated and incidence trends were modeled using Joinpoint. Results: 4367 lymphoid neoplasms were diagnosed in the Girona province. The ASRE for overall lymphoma was 37.1 (95\% CI: 36.0; 38.2), with a marked male predominance in almost all subtypes. During 1996-2015, incidence trends remained stable for broader lymphoma categories. According to our predictions, 17,950 new cases of LNs will be diagnosed in Spain in 2020.

Conclusions: This 'real-world' data will provide valuable information to better inform etiological hypotheses and plan future health-care services.
\end{abstract}

\section{Introduction}

Lymphoid neoplasms (LNs) are a heterogeneous group of hematological malignancies presenting diverse etiology, presentation and outcomes [1]. Changing classification schemes [i.e. the Working Formulation (WF-1982), the Revised European-American Lymphoma (REAL, 1994) and the World Health Organization (WHO, 2001, 2008 and 2016) classifications] and scarcity of subtype-specific epidemiological data hamper international comparisons. The aim of this study was to describe incidence patterns of LNs in the Girona province (Spain) (1996-2015) according to the WHO 2008 classification, and to predict the number of LNs in Spain during 2020.

\section{Material and methods}

Data were extracted from the population-based Girona cancer registry, covering a population of 738,976 inhabitants in 2015. Incident cases were registered using the International Classification of Diseases for Oncology, third edition (ICD-O-3) and grouped according to the WHO 2008 classification scheme. Age-adjusted incidence rates to the European standard population (ASRE) were estimated and Joinpoint regression modeling was used to examine temporal trends in agestandardized rates. Sex- and age-specific projections of cases in Spain in 2020 were estimated, based on the National Statistics Institute of Spain projections of population.

\footnotetext{
Abbreviations: ASRE, age-adjusted incidence rate to the European population; APC, annual percent change; ICD-O-3, International Classification of Diseases for Oncology, third edition; LN, lymphoid neoplasm; WHO, World Health Organization

* Corresponding author at: Campus de Montilivi, Carrer de la Universitat de Girona 10, 17003 Girona, Spain.

E-mail address: martasolans@gmail.com (M. Solans).
} 


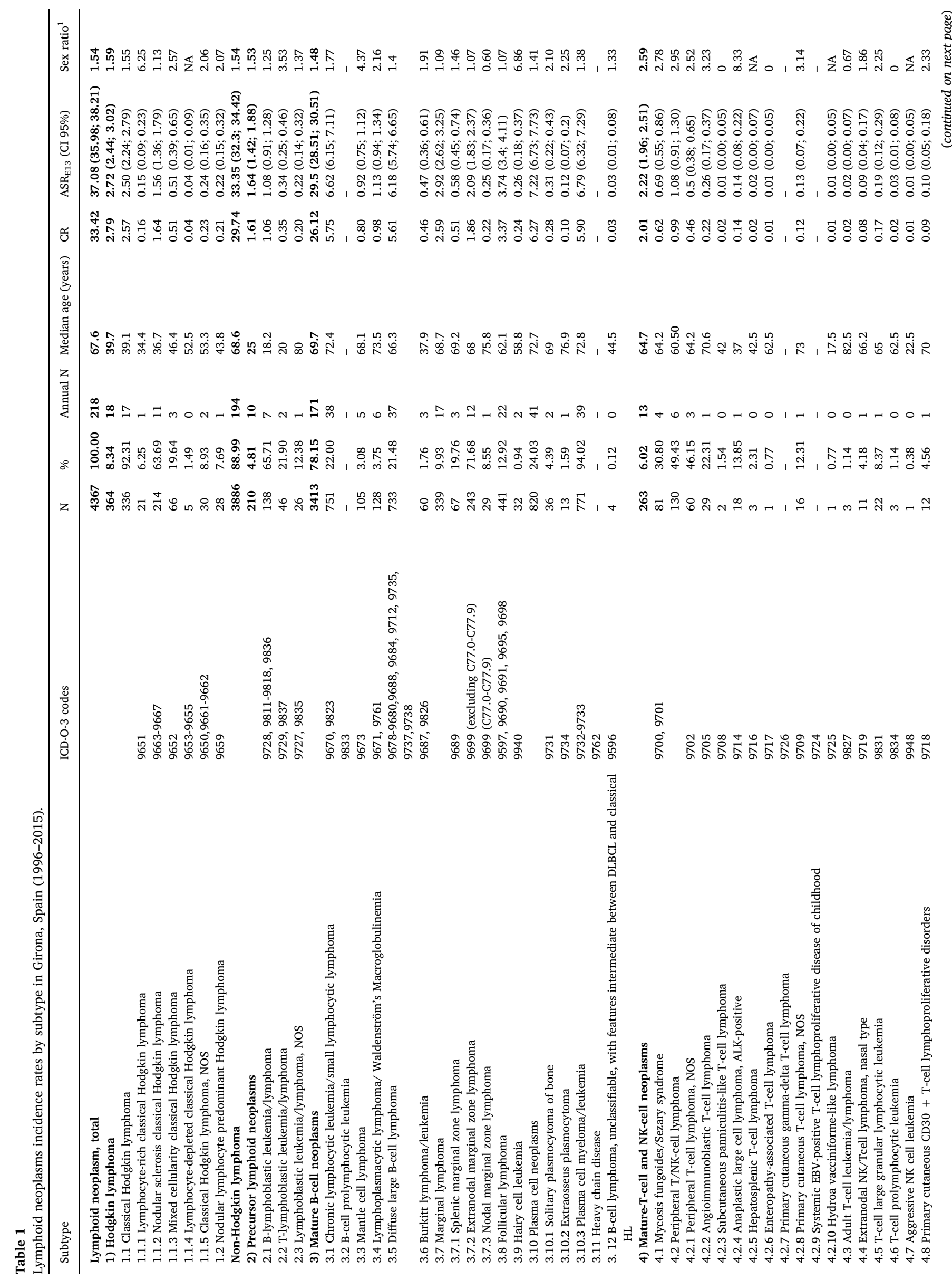


Table 2

Trends in lymphoid neoplasms incidence rates by major subtypes in Girona, Spain (1996-2015).

\begin{tabular}{llll}
\hline & Period & APC & CI 95\% \\
\hline Lymphoid neoplasms & $1996-2015$ & 0.1 & $(-0.6 ; 0.8)$ \\
Hodgkin lymphoma & $1996-2015$ & 0.7 & $(-1.4 ; 2.9)$ \\
Precursor lymphoid neoplasms & $1996-2015$ & -1.5 & $(-4.1 ; 1.2)$ \\
Mature B-cell neoplasms & $1996-2015$ & 0.3 & $(-0.5 ; 1.2)$ \\
$\quad$ Chronic lymphocytic leukemia/small & $1996-2015$ & -0.1 & $(-1.4 ; 1.3)$ \\
$\quad$ lymphocytic lymphoma & & & \\
$\quad$ Mantle cell lymphoma & $1996-2015$ & 1.8 & $(-2.8 ; 6.6)$ \\
$\quad$ Lymphoplasmacytic lymphoma/ & $1996-2015$ & 1.2 & $(-1.3 ; 3.9)$ \\
$\quad$ Waldenström's macroglobulinemia & & & \\
$\quad$ Diffuse large B-cell lymphoma & $1996-2015$ & 0.5 & $(-1.1 ; 2.0)$ \\
$\quad$ Burkitt lymphoma/leukemia & $1996-2015$ & -3.3 & $(-7.9 ; 1.6)$ \\
$\quad$ Marginal lymphoma & $1996-2015$ & 0.4 & $(-0.9 ; 1.7)$ \\
$\quad$ Follicular lymphoma & $1996-2015$ & 1.2 & $(-1.2 ; 3.6)$ \\
$\quad$ Plasma cell neoplasms & $1996-2015$ & 0.3 & $(-1.4 ; 1.9)$ \\
Mature T-cell and NK-cell neoplasmns & $1996-2015$ & 1.7 & $(-1.9 ; 5.6)$ \\
Lymphoid neoplasms, NOS & $1996-2015$ & -7.4 & $(-11.2 ;-3.3)$ \\
\hline
\end{tabular}

APC, annual percent change; NOS, not otherwise specified.

Calculated only for major lymphoma categories and most incident mature B-cell neoplasms.

* p-value $<0.05$.

\section{Results}

4367 LNs incident cases ( $59.7 \%$ men) were diagnosed in the Girona province. Among them, 364 (8.3\%) were Hodgkin-lymphoma, 210 (4.8\%) precursor lymphoid neoplasms, 3413 (78.2\%) mature B-cell neoplasms, 263 (6.0\%) mature T-cell and NK-cell neoplasms, and 117 (2.7\%) lymphoid neoplasms not otherwise specified (NOS). Detailed information can be found in Table 1. In brief, the median age at diagnosis ranged from 25 to 77.9 years according to the major LNs subtypes. Overall in both sexes, the top five most frequent entities were: plasma cell neoplasm (820), chronic lymphocytic leukemia/small lymphocytic lymphoma (751), diffuse large B-cell lymphoma (733), follicular lymphoma (441) and marginal lymphoma (339). The LNs crude rate (CR) was 33.4 and ASRE was 37.1 (95\% confidence interval (CI): 36.0; 38.2), 45.7 (95\% CI: 43.9; 47.6) in men and 29.7 (95\% CI: 28.4; 31.1) in women. There was a marked male predominance; the incidence sex ratio (male/female) ranged from 1.1 for follicular and marginal lymphomas to 6.9 for hairy cell leukemia.

During 1996-2015, no statistically significant variations in incidence trends were found for LNs [annual percentage change (APC): 0.1 (95\% CI: $-0.6 ; 0.8)$ ], nor for broader lymphoma categories (Table 2). Only the LNs NOS subtype category decreased, evidencing the improved diagnostic specificity for these malignancies during the last years.

According to our predictions, 17,950 new cases of LNs will be diagnosed in Spain in 2020, of which 1264 cases will be Hodgkin lymphoma, 738 precursor lymphoid neoplasms, 14,361 mature B-cell neoplasms (i.e. 3248 chronic lymphocytic leukemia/small lymphocytic lymphoma, 445 mantle cell lymphoma, 544 lymphoplasmacytic lymphoma/Waldenström's macroglobulinemia, 3025 diffuse large B-cell lymphoma, 221 Burkitt lymphoma/leukemia, 1394 marginal lymphoma, 1811 follicular lymphoma, 129 hairy cell leukemia, and 3531 plasma cell neoplasms), 1067 mature T-cell and NK-cell neoplasms and 521 LNs NOS (data not shown).

\section{Discussion}

This paper presents epidemiological data of LNs in the province of Girona (Spain) during a 20-year period. Incidence rates for the most frequent subtypes were in accordance with those reported in France (1980-2009) [2], United Kingdom (2004-2014) [3], Europe (2000-2002) [4], and generally lower in comparison to those from the 
United States (projected incidence in 2016) [5] or Australia (1982-2006) [6]. Regarding specific entities, we reported higher rates of mantel cell lymphoma (ASRE $=0.92$ ), to the date only linked to few genetic and environmental risk factors (i.e. atopy, allergy or farm life) [7], which merit further research. In the same vein, Burkitt lymphoma/ leukemia incidence (ASRE $=0.47$ ) was particularly high in our region, a pattern largely reported in other Southern European regions, yet unexplained by well-stablished etiological factors [8]. Similarly, we reported higher rates of chronic lymphocytic leukemia/small lymphocytic lymphoma (ASRE $=6.62$ ), mainly attributable to a higher completeness of our cancer registry for this entity rather than to a real peak of incidence in our region. Chronic lymphocytic leukemia - as well as Waldenström's macroglobulinemia - have been shown to be underreported by cancer registries given that they are prone to be indolent and their diagnosis is not based on tissue pathology [9]. To ensure a complete coverage of all chronic lymphocytic leukemia cases in our region, we recently performed review of alternative sources of information (e.g. flow cytometry and hematologists databases) and evidenced a $18.2 \%$ of underreported cases during 1998-2013 [10]. Nonspecialized cancer registries thus, should make additional efforts to ensure the surveillance of these malignancies is entirely accurate.

Incidence trends are broadly consistent with those reported for other Western countries, which describe a steady increase in the incidence of LNs during the 80 's and a stabilization in the late 90 's $[5,6,11]$. Furthermore, in the United States, even a slight decline in incidence rates by 2000 has been reported [5], which has been widely related to a decrease in AIDS incidence, but also suggested to reflect coding changes in 2001, when the WHO classification system was first published [12]. Despite concordance between ICD-O-3, which incorporates the WHO Classification, and cases originally diagnosed and coded under previous systems is generally high [13], several changes can be rarely overcame. For example, the 2008 International Workshop on chronic lymphocytic leukemia, changed the definition of the disease by requiring an absolute B-cell count of 5000 cells/ $\mu \mathrm{L}$ rather than the previous absolute lymphocyte count of 5000 cells/ $\mu \mathrm{L}$, causing many former Rai stage 0 cases to be reclassified as monoclonal B-cell lymphocytosis, a pre-malignant disorder not recorded by cancer registries [14]. Overall, our results have to be interpreted cautiously since the numerous changes in the classification of LNs over time could result in errors of diagnosis or coding translation, particularly during the earlier period.

The number of expected lymphoma cases depicts accurately the cancer burden of LNs in Spain, which lacks of a cancer registry with national coverage, but data on specific subtypes should be interpreted with caution. Some WHO LNs subtypes are extremely rare, making estimates less robust. However, overall, these results are interesting for clinicians and public health in evaluating the cost of management and new treatments for these pathologies.

In 2016, a new WHO manual was released [15] but the available surveillance data are through 1996-2015 and do not reflect these updates. The capability of registries to recode previous cases to these new schemes will be limited because many require additional molecular or clinical data [5]. However, changes to the 2008 classification are relatively small and would not be expected to change our conclusions. The impact of these changes will be unclear until next years, when they become adopted by pathologists and new entities routinely distinguished in clinical practice.

\section{Conclusions}

In conclusion, this study describes in detail the incidence of LNs categorized by 2008 WHO subtype in a large population-based cohort. This has not been performed previously for Spain, and complements the subtype-specific analyses published for Europe [2-4], the United States [5], and Australia [6]. This 'real-world' data will provide valuable information to better inform etiological hypotheses and plan future health-care services.

\section{Author contribution}

- Study conception and design: RMG, MSo

- Acquisition of data: RMG, AF, IG, JMR, AB, NK, JB

- Analysis of data: AF, DM

- Interpretation of data: MSo, RMG, AF, DM, CA, MSa

- Manuscript writing: MSo

- Critical review of the article and final approval of the article: all authors

\section{Funding}

This work was supported by the Spanish Ministry of Health (FIS PI15/00966) and by CIBERESP (PhD contract to MS).

\section{Conflict of interest}

None.

\section{References}

[1] S.H. Swerdlow, E. Campo, N.L. Harris, E.S. Jaffe, S.A. Pileri, H. Stein, J. Thiele, J. Vardiman, International Agency for Research on Cancer (Ed.), WHO Classification of Tumours of Haematopoietic and Lymphoid Tissues, 2008, p. 439 Lyon, France.

[2] M. Dandoit, M. Mounier, J. Guy, T. Petrella, S. Girard, R.-O. Casasnovas, et al., The heterogeneity of changes in incidence and survival among lymphoid malignancies in a 30-year French population-based registry, Leuk. Lymphoma 56 (4) (2015) 1050-1057.

[3] A. Smith, S. Crouch, S. Lax, J. Li, D. Painter, D. Howell, et al., Lymphoma incidence, survival and prevalence 2004-2014: sub-type analyses from the UK's Haematological Malignancy Research Network, Br. J. Cancer 112 (9) (2015) 1575-1584.

[4] M. Sant, C. Allemani, C. Tereanu, R. De Angelis, R. Capocaccia, O. Visser, et al., Incidence of hematologic malignancies in Europe by morphologic subtype: results of the HAEMACARE project, Blood 116 (19) (2010) 3724-3734.

[5] L.R. Teras, C.E. DeSantis, J.R. Cerhan, L.M. Morton, A. Jemal, C.R. Flowers, US lymphoid malignancy statistics by World Health Organization subtypes, CA Cancer J. Clin. 2016 (September (12)) (2016), https://doi.org/10.3322/caac.21357 [Epub ahead of print].

[6] M.T. van Leeuwen, J.J. Turner, D.J. Joske, M.O. Falster, P. Srasuebkul, N.S. Meagher, et al., Lymphoid neoplasm incidence by WHO subtype in Australia 1982-2006, Int. J. Cancer 135 (9) (2014) 2146-2156.

[7] K.E. Smedby, J.N. Sampson, J.J. Turner, S.L. Slager, M. Maynadie, E. Roman, et al., Medical history, lifestyle, family history, and occupational risk factors for mantle cell lymphoma: the InterLymph Non-Hodgkin Lymphoma Subtypes Project, J. Natl. Cancer Inst. Monogr. 2014 (48) (2014) 76-86.

[8] M.I. Izarzugaza, E. Steliarova-Foucher, M.C. Martos, S. Zivkovic, Non-Hodgkin's lymphoma incidence and survival in European children and adolescents (19781997): report from the Automated Childhood Cancer information System project, Eur. J. Cancer 42 (13) (2006) 2050-2063.

[9] I. Turesson, M.S. Linet, M. Bjorkholm, S.Y. Kristinsson, L.R. Goldin, N.E. Caporaso, et al., Ascertainment and diagnostic accuracy for hematopoietic lymphoproliferative malignancies in Sweden 1964-2003, Int. J. Cancer 121 (10) (2007) 2260-2266.

[10] M. Solans, G. Osca-Gelis, R. Comas, J.M. Roncero, D. Gallardo, R. Marcos-Gragera, et al., Challenges in assessing the real incidence of chronic lymphocytic leukemia: 16 years of epidemiological data from the province of Girona, Spain, Cancer Causes Control 29 (3) (2018) 379-382.

[11] P. Adamson, F. Bray, A.S. Costantini, M.-H. Tao, E. Weiderpass, E. Roman, Time trends in the registration of Hodgkin and non-Hodgkin lymphomas in Europe, Eur. J. Cancer 43 (2) (2007) 391-401.

[12] M.S. Shiels, E.A. Engels, M.S. Linet, C.A. Clarke, J. Li, H.I. Hall, et al., The epidemic of non-Hodgkin lymphoma in the United States: disentangling the effect of HIV, 1992-2009, Cancer Epidemiol. Biomarkers Prev. 22 (6) (2013) 1069-1078.

[13] C.A. Clarke, D.M. Undurraga, P.J. Harasty, S.L. Glaser, L.M. Morton, E.A. Holly, Changes in cancer registry coding for lymphoma subtypes: reliability over time and relevance for surveillance and study, Cancer Epidemiol. Biomarkers Prev. 15 (4) (2006) 630-638.

[14] T.G. Call, A.D. Norman, C.A. Hanson, S.J. Achenbach, N.E. Kay, C.S. Zent, et al., Incidence of chronic lymphocytic leukemia and high count monoclonal B-cell lymphocytosis using the 2008 guidelines, Cancer 120 (13) (2014) 2000-2005.

[15] S.H. Swerdlow, E. Campo, S.A. Pileri, N.L. Harris, H. Stein, R. Siebert, et al., The 2016 revision of the World Health Organization (WHO) classification of lymphoid neoplasms, Blood (127) (2016) 2375-2390. 